\title{
Childhood psychological abuse and neglect to college freshmen' automatic negative thoughts: Mediating roles of social support
}

\author{
Zengyan Yu, Wei Fu \\ School of mental health, Qiqihar Medical University, Qigihar Heilongjiang, 161006,China
}

\begin{abstract}
Keywords: Childhood psychological abuse and neglect, Automatic negative thoughts, Social support, Cross-sectional survey.
\end{abstract}

\begin{abstract}
Objective : To explore the relationship of college freshmen' automatic negative thoughts, social support, childhood psychological abuse and neglect. Methods: 310freshmen from two colleges of Heilongjiang Province were surveyed with the Child Psychological Abuse and Neglect Scale (CPANS),Automatic Thoughts Questionnaire (ATQ) and Social Support Rating Scale(SSRS).Rating Scale(SSRS).Results: There were significant difference son gender, family and maternal structure educational level with scores of child hood psychological abuse and neglect $(\mathrm{P}<0.01)$; The testing of mediating effects showed that childhood psychological abuse and neglect had both direct and indirect effects on automatic negative thoughts, with the direct effect as the main effect. Conclusion: Childhood psychological abuse has direct effects on automatic negative thoughts, but also has indirect impacts through social support. The mediating roles of social support suggest salient leverage points for easing negative cognitive thinking with a certain extent through social support in college freshmen.
\end{abstract}

\section{Introduction}

In recent years, because of the development of human civilization and the change of medical model, child psychological abuse and neglect have gradually become the international focus issues. The proof of the negative effect caused by psychological abuse and neglect in childhood constantly emerges, which indicates that after the physical abuse and sexual abuse having been prohibited, psychological abuse and neglect are becoming more and more common for encroaching on the healthy growth of teenagers.

Domestic and overseas researchers made a large number of researches based on Beck's mental disorder cognitive theory, the results showed that as a kind of early traumatic experience of an individual, psychological abuse in one's childhood affects his/her cognitive function formation and development, then leads to the depression, anxiety, self-abasement, interpersonal sensitivity and social maladjustment in the adult stage. For example, E.Calvete's ( 2013 ) $\operatorname{search}^{[1]}$ found that children who suffered psychological abuse are easy to form the pictorial features of autonomic damage and guided by others, these pictorial features caused the generation of individual's depression and social anxiety. Lisa ReingoldMelmed (2012) thesis $^{[2]}$ showed that male teenagers' cognitive type has influence on the physical abuse and depression. Domestic scholars like Liu Aishu (2013) also found the medical function of automatic thinking of child psychological abuse and depression, proposed that junior high school students' depressive state can be improved from the cognitive aspects.

Above researches showed the medical function of cognitive features on child psychological abuse and neglect for individual's later negative impact, and propose to reduce the negative effect caused by psychological abuse and neglect through changing cognition, while how to improve the adverse cognition caused by child psychological abuse and neglect still lack corresponding theory and practice. In addition, according to the Relational regulation theory, RRT of Lakey (2011), good social 
support can effectively adjust all kinds of negative thinking type level, while in the negative cognitive thinking like negative automatic thoughts, hopelessness, perfectionism and dysfunctional attitudes, negative automatic thoughts largely influenced by social factors ${ }^{[4]}$. Therefore, this research choose new college students as study objects, propose the assumption that the medical function system of social support on child psychological abuse and neglect and the negative automatic thoughts, wants to provide some guidelines for the effective improvement of teenagers' negative cognitive thoughts and improve college students' adaptive ability.

\section{Objects and Methods}

\section{Objects}

Use convenient sampling, take freshmen of 2013 grade of two colleges in Heilongjiang province as study objects, hand out 310 questionnaires, gain 294 back, recovery efficiency is 94.8\%. Among which, 150 male students, 44 female students, average age is $19 \pm 1$ years old.

\section{Tools}

(Child Psychological Abuse and Neglect Scale, CPANS)[5]

Total has 31 items, use 5 level score. Including two subscales of psychological abuse and psychological neglect, and six dimensions of blame, threaten, interfere, emotional neglect, education neglect and body neglect. The internal consistency reliability of the scale in this research is 0.85 .

The Automatic Thoughts Questionnaire ATQ[6]

Total has 30 automatic negative thoughts to express the internal self-description of cognitive experience. Use 1-5 level score, its scores and depression degree is positive related. The internal consistency reliability of the scale in this research is 0.94 .

Social Support Rating Scale[6]

Total has 10 items, including three dimensions of objective support, subjective support and social support. It has good reliability and validity. The internal consistency reliability of the scale in this research is 0.71 .

\section{Statistical methods}

After collecting all the questionnaires, make initial clear up for the questionnaire and calculate its recovery efficiency. Then use SPSS13.0 to make data entry, mainly use statistical methods of $t$ inspection, variance analysis, Pearson correlation analysis and linear-regression analysis. At last, use AMOS17 software to make structural equation model analysis and test the medical effect of social support.

\section{Result}

Difference of the college freshmen scales on sexual, family structure and mother's education background

Use $t$ test to check the difference of college freshmen's child psychological abuse and neglect, automatic thoughts and social support on the demographic variables, found out that the difference of child psychological abuse and neglect, automatic thoughts and social support on family structure level has statistical meaning $(\mathrm{P}<0.05)$, but on the comparison of sexual level, only the child psychological abuse and neglect, automatic thoughts has statistical meaning $(\mathrm{P}<0.01)$, see table 1 . In addition, use variance analysis found that child psychological abuse and neglect, automatic thoughts on mother's education background also has statistical meaning ( $\mathrm{F}(3,190)=4.77, \mathrm{P}=0.003$; $\mathrm{F}(3,190)=4.35, \mathrm{P}=0.003)$, later the test analysis showed that mother's education background on literacy and other difference also has statistical meaning $(\mathrm{P}<0.01)$. 
Table 1. Difference of College Freshmen Scale on Sexual and Family Structure $\left(\bar{x}_{ \pm} \mathrm{S}\right)$

\begin{tabular}{|c|c|c|c|c|c|c|}
\hline \multirow[b]{2}{*}{ Scale } & \multicolumn{2}{|c|}{ Sexual } & & \multicolumn{2}{|c|}{ Family Structure } & \multirow[b]{2}{*}{$\mathrm{t}$} \\
\hline & Male & Female & & Complete & Not Complete & \\
\hline Psychological abuse and neglect & $32.69 \pm 13.63$ & $27.33 \pm 14.25$ & $2.68 * *$ & $28.82 \pm 13.54$ & $39.13 \pm 15.41$ & $-3.43 * *$ \\
\hline Automatic thoughts & $64.88 \pm 20.58$ & $57.73 \pm 15.55$ & $2.71^{* *}$ & $60.32 \pm 17.88$ & $69.17 \pm 22.08$ & $-2.20 *$ \\
\hline Social support & $27.78 \pm 5.02$ & $28.57 \pm 4.08$ & -1.21 & $28.58 \pm 4.44$ & $25.21 \pm 4.65$ & $3.46^{* *}$ \\
\hline
\end{tabular}

Note: $* \mathrm{P}<0.05$, ** $\mathrm{P}<0.01$, *** $\mathrm{P}<0.001$, the following is the same.

\section{Correlation of college freshmen scores}

Psychological abuse and neglect is positive related with automatic thoughts, automatic thoughts are negative related with social support, psychological abuse and neglect is negative related with social support, see table 2 .

Table 2. Correlation of college freshmen scores ( $r$ )

\begin{tabular}{cccc}
\hline Scores & $\bar{x}_{ \pm \mathrm{S}}$ & Psychological abuse and neglect & Automatic thoughts \\
\hline Psychological abuse and neglect & $30.09 \pm 14.15$ & & \\
Automatic thoughts & $61.42 \pm 18.62$ & $0.49^{* *}$ & $-0.43^{* *}$ \\
Social support & $28.16 \pm 4.59$ & $-0.38^{* *}$ & \\
\hline
\end{tabular}

\section{The medical effect of social support on psychological abuse and neglect and automatic thought}

Use structural equation model technology to construct model, make theoretical hypothesis testing analysis, like picture 1 . Result showed that all matching indexes of structural equation model are good (see table 3), it indicates that data and structural model matching well, setting is reasonable. According to the medical effect testing method of Wen Zhonglin (2004), we can think that social support has partial medical function on the influence process of psychological abuse and neglect on automatic thoughts. Therefore, among the influence of child psychological abuse and neglect on college students' automatic thought, including psychological abuse and neglect on automatic thought, the directly influence accounts $54.1 \%$, indirect influence of social support accounts $8 \%$, social support accounts $12.9 \%$.

Table 3. Matching indexes of model construction

\begin{tabular}{ccccccccc}
\hline$\chi^{2}$ & $v$ & $\chi 2 / v$ & RMSEA & GFI & AGFI & CFI & IFI & NFI \\
\hline 25.689 & 12 & 2.141 & 0.077 & 0.968 & 0.905 & 0.966 & 0.971 & 0.946 \\
\hline
\end{tabular}




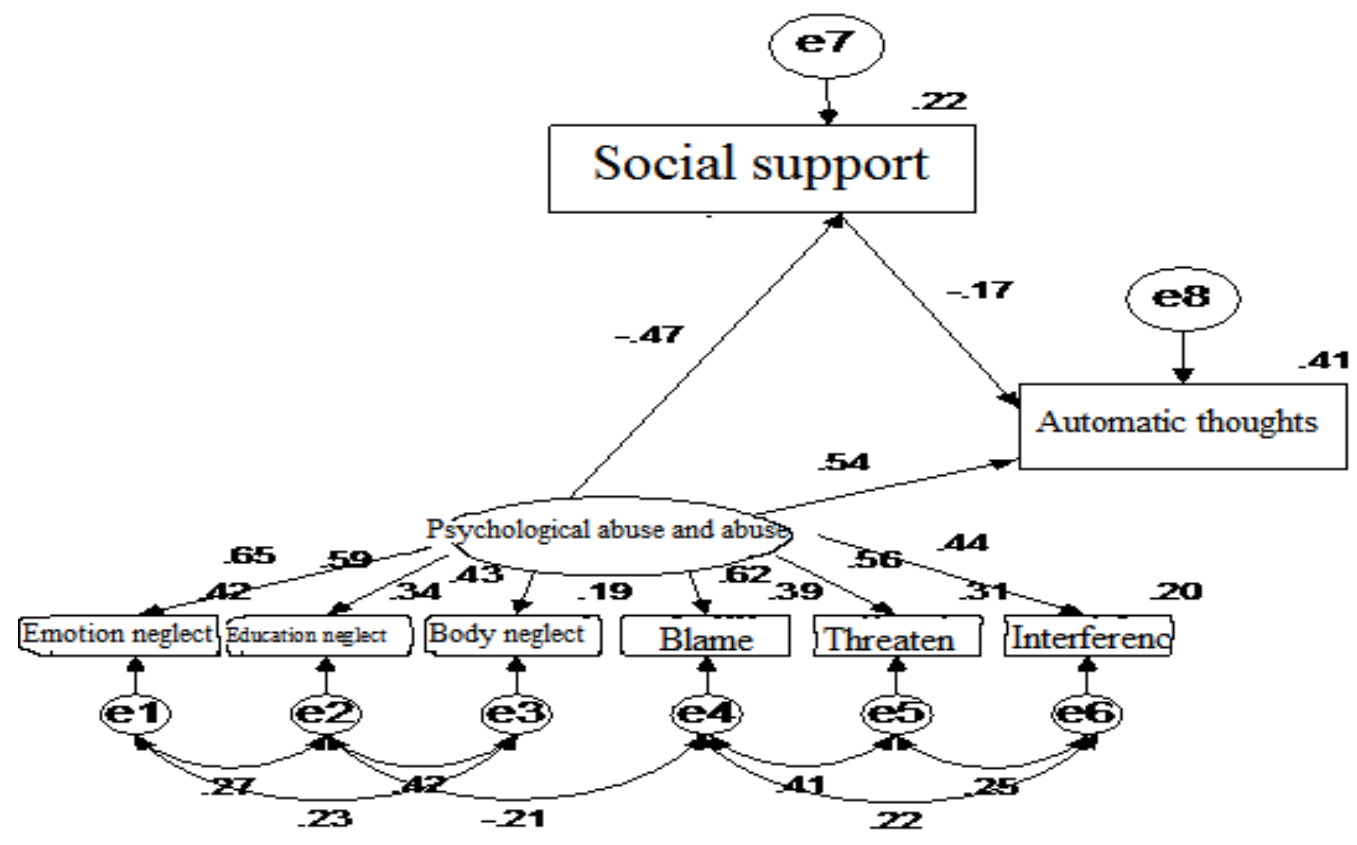

Fig. 1. Diagrammatic figure of social support as the mediating variable of psychological abuse and neglect and automatic thoughts

\section{Discussion}

This research found that psychological abuse and neglect of female students is obviously higher than females, it might be originated from the more strict education on males according to traditional family education concept, but whether there is sexual difference of the occurrence rate of psychological abuse and neglect is not sure, research showed that ${ }^{[7]}$, core family suffers less from emotion abuse. It is thus clear that incomplete family might be one of the reasons for psychological abuse and neglect.

Structural equation model in this research showed that child psychological abuse and neglect has direct function on college freshmen's negative automatic thoughts, that is to say, child psychological abuse and neglect is easy to cause negative cognitive thoughts thus to cause individual's depression and anxiety.

While child psychological abuse and neglect is mainly directly influence college freshmen' s automatic thoughts, though part of the social part has obvious effect, the reason for its exist might be the small quantity of tested object, who have concerns from the social expectation when fill the psychological abuse and neglect questionnaire and automatic thought thus they did not fill it truly. These problems should be solved through adding sample quantity and type in the future research, and try to gain more information on child psychological abuse and neglect through various ways, thus to improve the reliability and validity of research.

\section{Acknowledgement}

This paper is the humanities and social sciences project of the Heilongjiang provincial education department (Project No.: 12542341) .

\section{References}

[1] Calvete E. Emotional abuse as a predictor of early maladaptive schemas in adolescents:Contributions to the development of depressive and social anxiety symptoms, Child Abuse \& Neglect,2013. 
[2] Melmed L R. Cognitive style as a mediator between parental psychological maltreatment and depression in adolescent boys, Thesis,2012.

[3] Lakey B, Tanner S M. Social Influences in Negative Thinking and Affect, Cognitive Therapy and Research,2013,37(1):160-172.

[4] Deng Yunlong, Pan Chen, Tang Qiuping and so on. Primary Construction of the Scale of Child Psychological Abuse and Neglect, Chinese Behavioral Medical Science,2007,16(2):175-177.

[5] Wang Xiangdong, Wang Xilin, Mahong. Mental Health Assessment Scale, Beijign: Chinese Psychological Magazine, 1999,127-217.

[6] Brassard M R, Donovan K L. Defining psychological maltreatment, Child abuse and neglect: Definitions, classifications, and a framework for research, Feerick J F, Knutson P K, Baltimore: Paul H.Brookes,2006.,151-197. 